\title{
Multi-Wavelength AGN from Ground and Space, from Far-IR to High-Energy
}

Daniel Stern

California Institute of Technology, USA

Email: daniel.k.stern@jpl.nasa.gov

\begin{abstract}
I will discuss multi-wavelength AGN studies, with a focus on mid-IR and radio selected obscured AGN. Obscured AGN, which are robustly identified across the full sky by WISE, are the dominant AGN population. I will discuss several aspects of the mid-IR obscured AGN population, ranging from detailed studies of extreme sources, the so-called WISE ultraluminous 'hot dust-obscured galaxy' or 'hot DOG' sample, as well as more general studies comparing obscured and unobscured AGN identified in wide-area surveys.
\end{abstract}

